\title{
Extending the Bayesian Classifier to a Context-Aware Recommender System for Mobile Devices
}

\author{
Toon De Pessemier, Tom Deryckere, and Luc Martens \\ Wireless and Cable Group, Dept. of Information Technology (INTEC) \\ IBBT - Ghent University \\ Ghent, Belgium \\ tdpessem@intec.ugent.be,tderycke@intec.ugent.be, luc.martens@intec.ugent.be
}

\begin{abstract}
Mobile devices that are capable of playing Internet videos have become wide-spread in recent years. Because of the enormous offer of video content, the lack of sufficient presentation space on the screen, and the laborious navigation on mobile devices, the video consumption process becomes more complicated for the end-user. To handle this problem, people need new instruments to assist with the hunting, filtering and selection process. We developed a methodology for mobile devices that makes the huge content sources more manageable by creating a user profile and personalizing the offer. This paper reports the structure of the user profile, the user interaction mechanism, and the recommendation algorithm, an improved version of the Bayesian classifier that incorporates aspects of the consumption context (like time, location, and mood of the user) to make the suggestions more accurate.
\end{abstract}

Keywords- recommendation system; user-generated content; mobile device; folksonomy; context awareness

\section{INTRODUCTION}

With the growing capability and popularity of mobile phones and smart phones, users have become very enthusiastic in watching videos through their pervasive mobile devices. Moreover, increasingly more mobile devices provide "anytime, anywhere" Internet access. The combination of these two developments makes the content of the web 2.0 video sharing sites [1], like YouTube [2] and Vimeo [3], available on the move.

Unfortunately, these new services complicate the video selection process for the end-user who can only consume a small fraction of the overwhelming quantity of video content. Furthermore, a lot of user-generated videos are annoying, irrelevant, or not in the field of interest of the viewer. Most video sites use a keyword-based search tool to address these problems. Nevertheless, this rudimentary search tool is not capable to weed out irrelevant content. A second filtering based on user ratings or consumption patterns can assist. Community websites like Digg [4] effectively filter their content based on the interaction behavior of the community. However, these techniques require a broad base of user feedback before they can make a reasonable suggestion. Moreover, the enthusiasts of niche content are not taken into account, since only popular content will be favored by the majority of the community.
As a consequence, there is still a need for better tools and technologies to search large collections of multimedia data and to deliver the right content according to the current needs and preferences. Recommendation systems try to solve this problem by creating and updating a user profile in the background, followed by filtering and recommending content according to the gathered preference information.

The remainder of this paper is organized as follows: Section 2 provides an overview of previous research work related to personal recommendations. In Section 3, we present the architecture and the features of our developed recommendation application. The proposed algorithm, the Bayesian classifier enriched with the consumption context, is discussed in detail in Section 4. Next, Section 5 further elaborates on the recommendation algorithm by providing some optimizations. Finally, we offer a brief conclusion and point out future work in Section 6.

\section{RELATED WORK}

The overabundance of content and the related difficulty to discover interesting content items, is already addressed in several contexts. Online shops, like Amazon, apply collaborative filtering to personalize the online store according to the needs of each customer [5]. The items that customers purchase and explicitly rate will represent their interests and are used for future recommendations [6].

Netflix [7] is an online, mail-based, DVD rental service to customers in the United States. After renting a movie, customers may enter their rating of the movie into the Netflix database via the website, using a concrete score from 1 to 5 . In October 2006, Netflix published a large dataset and began a competition to find better recommendation systems. Many research groups are competing to find the best movie recommendation algorithm in this context.

The introduction of digital television entails an increase in the number of available TV channels and the coupled information overload. Consequently, new standards to describe this editorial content (e.g., TV-Anytime [5]) and advanced electronic program guides, which simplify the navigation and searching of TV programs, become necessary [9]. Several personalized TV guide systems which filter and recommend TV programs according to the user's preference information, are already developed for set-top boxes [10] and personal digital recorders [11][12].

We have lately witnessed the generation of loads of usergenerated content on the web: photos, videos, bookmarks, 
etc. The web 2.0 applications apply more pragmatic approaches (e.g., tagging) to annotate content than the traditional classification systems, like TV-Anytime. Such a metadata description which is the contribution of the whole community, is called a folksonomy and has become very popular on the web around 2004. Since every user will tag in a different manner and use other synonyms [13], recommendation systems for user-generated content have to cope with an extra difficulty. Several recommendation systems for user-generated content are described in literature [14], however our proposed system distinguishes itself by providing context-aware suggestions on the mobile platform.

Because people generally restrict their viewing time on the small displays of mobile devices and minimize the amount of interaction and navigation during the selection process, recommending content on mobile devices is a research problem with growing importance. The GroupLens [15] lab conducted research in a movie recommender system on a cell phone browser and showed that recommender systems can be valuable services on mobile devices [16]. Moreover, researchers are even attempting to combine summarization and personalization of video content on mobile devices to protect people from the profusion of content [17]. Our work goes beyond the existing recommendation systems for mobile devices by extending the offer with user-generated content and taking the consumption context into account.

\section{ARCHITECTURAL OVERVIEW OF THE RECOMMENDATION SYSTEM}

To deal with the profuse offer of user-generated content on mobile devices, we developed a recommendation algorithm which filters and suggests audio-visual content based on a personal user profile. As proof of concept, we incorporated the algorithm in a video application and implemented it on a Nokia N95 mobile device. This device that runs on Symbian OS 9.2, has a $3 \mathrm{G}$ and WiFi connection, 64MB RAM, a Dual ARM 11 processor that operates at 332 $\mathrm{MHz}$ processor and a 3D Graphics HW Accelerator. During the application development, special attention was paid to the usability and ease of navigation.

Figure 1 shows an architectural overview of the video recommendation application as well as the communication between mobile device, video server and recommendation server. End-users have the possibility to query the video content on their mobile device by a standard keyword-based search mechanism, just like on the YouTube website.

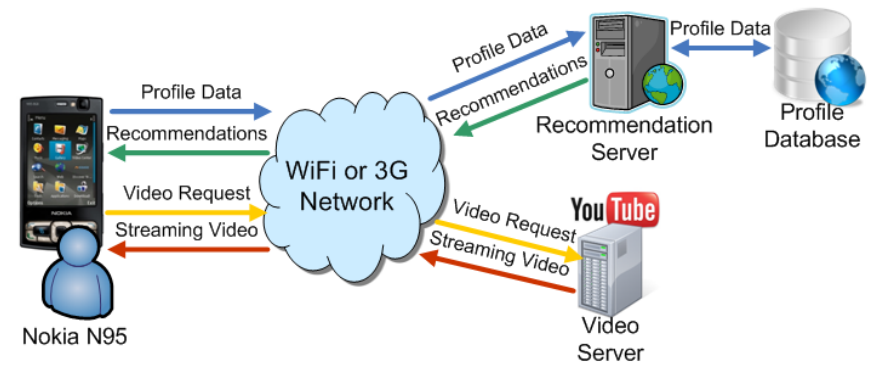

Figure 1. Architectural overview of the recommendation application.

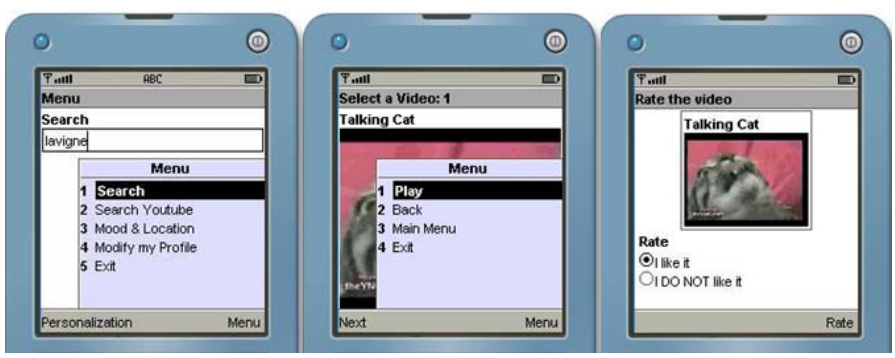

Figure 2. Screenshot of the mobile application: searching, consuming, and evaluating a content item.

The video selection mechanism triggers a request to the video server, which will stream the requested video via RTSP (Real Time Streaming Protocol). To provide the recommendation application with a broad range of videos, the YouTube content is retrieved via its Application Programming Interface (API).

Just after the video consumption, users can express their appreciation for the content item by specifying a binary rating. The mobile device sends this feedback (i.e. profile data) to the recommendation server, which will store this information in the profile database. By mining these content ratings, user preferences can be tracked, profiles can be created, and an additional application feature becomes available: receiving content suggestions based on the personal profile and the available context features (i.e. mood, location, time).

The recommendation server calculates these personal video suggestions based on the algorithm that is explained in Section 4 and sends these suggestions to the personal mobile device of the user. Afterwards, the user can select one of these video recommendations, which triggers a request to stream the selected video. Figure 2 displays some screenshots (created with an emulator) of the proposed application, which illustrates this searching, consumption, and rating process.

\section{THE BAYESIAN ClASSIFIER ENRICHED WITH THE CONSUMPTION CONTEXT}

Because navigation and interaction mechanisms on mobile devices are rather tiresome and laborious, we opted for a recommendation algorithm, which requires no or limited explicit user feedback. We decided upon the Bayesian classifier, computing the likelihood that the viewer will like or dislike a particular content item. The standard Bayesian classifier has already proved its efficiency as classification mechanism for content-based video suggestions in several previous research works [11][18].

\section{A. User Feedback}

The calculations are based on explicit user feedback, which consists of binary user appreciations (liked or disliked) for previously consumed content items. If an explicit feedback channel is not available, the Bayesian classifier has to rely on implicit feedback to gather user preferences [19]. This is often the case with personalized program guides for iDTV, which do not request to provide a rating [20][21]. The watching behavior of the user will be 
mapped to a binary appreciation value and fed to the Bayesian classifier. Liberating the user from the content evaluation process by relying on implicit feedback, might however lead to incorrect assumptions and a related decrease in algorithm performance.

\section{B. Consumption Context}

A context can be defined as a description of aspects of a situation (e.g., location, time, etc.) [22]. One challenge of mobile services is to make use of context information and exploit its changes. The rise of many context-aware applications for mobile devices confirms the interest in contextual information [23]. Investigating patterns in user behavior and the associated consumption context can deliver an insight into context dependent user habits and specific consumption behavior.

Driven by this insight, we enriched the recommendation algorithm with several aspects of the consumption context. Therefore, the mobile application offers users the possibility to select their current mood ('sad' or 'happy') and location ('on the move', 'at home', 'at work', or 'somewhere else') from a predefined list of possibilities. This information, completed with a timestamp, constitutes the consumption context and will be used as an extra information source of the algorithm. In an ideal world, the complete consumption context should be retrieved without any user interference. For example, the location of the user should be indentified through GPS coordinates. However, automatically retrieving the consumption context is a separate problem that lies beyond the scope of this research work, since particular aspects of the context such as the user's mood, are very difficult to retrieve without any user interference.

\section{The Enriched Bayesian Classifier}

The standard Bayesian classifier consists of a 2-class decision model, where a content item belongs to the class "liked", or the class "not liked". We will label these classes $\mathrm{C}^{+}$and $\mathrm{C}^{-}$respectively. The user profile, in the Bayesian context, is a collection of features together with a count of how many times each feature occurs in the set of positive and negative evaluated content items (i.e., in the classes $\mathrm{C}^{+}$ and $\mathrm{C}^{-}$) [21]. These features are characteristics of the content item and are typically metadata elements such as a category or a tag.

To incorporate context information, we had to extend the user profile with an additional dimension which specifies the context in which the user has consumed the content. An excerpt from an example user profile is shown in Figure 3. Users can specify their location and mood by selecting an option from a limited list in the application. Moreover, since timestamps are classified as 'morning', 'afternoon', or 'evening', the possible context situations are finite, which makes the algorithm calculations manageable.

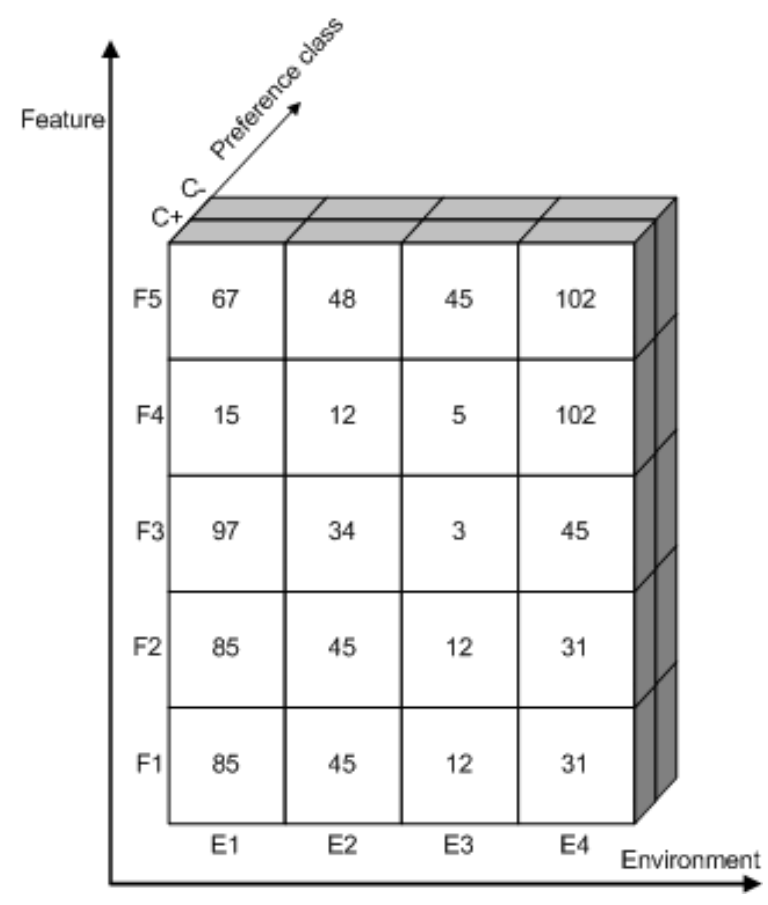

Figure 3. An excerpt from an example profile of the context-aware Bayesian classifier.

The traditional Bayesian classifier calculates the conditional probability, $\mathrm{P}(\mathrm{C}+\mid \mathrm{x})$, that the viewer will like an unseen content item, given its feature set (vector $\mathrm{x}$ ). Content items will be classified as "recommendations" if this probability is above a certain threshold. We extended the conditional probability with an extra condition, E, which indicates the environment or context of the consumption.

$$
\mathrm{P}\left(\mathrm{C}^{+} \mid \mathrm{x}, \mathrm{E}\right)
$$

Thus, (1) symbolizes the chance that an unseen content item will be in the class $\mathrm{C}+$, i.e., it will be liked by the user, given the feature set of the content item and the consumption environment of the user. The modus operandi to estimate this unknown probability is to convert this a posteriori probability in a computable a priori probability.

$$
P\left(C^{+} \mid x, E\right)=\frac{P\left(C^{+} \mid E\right) \cdot P\left(x \mid C^{+}, E\right)}{P(x \mid E)}
$$

In this equation, the denominator can be expanded, as follows:

$$
P(x \mid E)=P\left(C^{+} \mid E\right) \cdot P\left(x \mid C^{+}, E\right)+P\left(C^{-} \mid E\right) \cdot P\left(x \mid C^{-}, E\right)
$$

Through these conversions, rest us to calculate: $\mathrm{P}\left(\mathrm{C}^{+} \mid \mathrm{E}\right)$, $\mathrm{P}\left(\mathrm{x} \mid \mathrm{C}^{+}, \mathrm{E}\right)$, and the counterparts for the class $\mathrm{C}^{-}: \mathrm{P}\left(\mathrm{C}^{-} \mid \mathrm{E}\right)$ and $\mathrm{P}\left(\mathrm{x} \mid \mathrm{C}^{-}, \mathrm{E}\right)$. Furthermore, $\mathrm{P}\left(\mathrm{C}^{+} \mid \mathrm{E}\right)$ represents the probability that the viewer will like the content item if the consumption takes place at context environment $\mathrm{E}$, without the knowledge 
of any further information about the item. This likelihood can be estimated by the fraction of positive examples in that context.

$$
P\left(C^{+} \mid E\right)=\frac{k\left(C^{+}, E\right)}{k(E)}
$$

$\mathrm{k}(\mathrm{E})$ stands for the number of consumed items in the profile with a context equal to $\mathrm{E}$. This collection consists of a number of items which were appreciated, $\mathrm{k}\left(\mathrm{C}^{+}, \mathrm{E}\right)$, and a number of items which were not, $\mathrm{k}\left(\mathrm{C}^{-}, \mathrm{E}\right)$.

Analogously, the probability that the viewer dislikes the content item at a particular context is given by:

$$
P\left(C^{-} \mid E\right)=\frac{k\left(C^{-}, E\right)}{k(E)} .
$$

To estimate $\mathrm{P}\left(\mathrm{x} \mid \mathrm{C}^{+}, \mathrm{E}\right)$ and $\mathrm{P}\left(\mathrm{x} \mid \mathrm{C}^{-}, \mathrm{E}\right)$, we simplify the calculations by supposing that the elements of the feature set, $\mathrm{x}=\left\{\mathrm{f}_{1}, \mathrm{f}_{2}, \ldots, \mathrm{f}_{\mathrm{k}}\right\}$, are conditionally independent once the user's appreciation (and context) are known.

$$
\begin{gathered}
P\left(f_{1}, f_{2}, \ldots, f_{k} \mid C^{+}, E\right)=\prod_{i=1}^{k} P\left(f_{i} \mid C^{+}, E\right) \\
P\left(x \mid C^{+}, E\right)=\prod_{i=1}^{k} P\left(f_{i} \mid C^{+}, E\right) \prod_{i=k+1}^{n}\left(1-P\left(f_{i} \mid C^{+}, E\right)\right)
\end{gathered}
$$

$f_{1}$ till $f_{k}$ are features of the vector $x$, whereas $f_{k+1}$ till $f_{n}$ are all the features which are not part of $\mathrm{x}$. An analogous expression can be formulated for the class $\mathrm{C}^{-}$.

Although the assumption is violated in many fields of application, this simplification is commonly performed in Bayesian classifiers [11]-[19]. In spite of this incorrect assumption, Bayesian classifiers still have a surprisingly good performance. The key to this finding lies in the distinction between classification and probability estimation: correct classification can be achieved even when the probability estimates used contain large errors [24].

The portion of consumed items in the class $\mathrm{C}^{+}$and with context $E$ that are characterized by the feature $\mathrm{f}_{\mathrm{i}}$, can be an estimation for $\mathrm{P}\left(\mathrm{f}_{\mathrm{i}} \mid \mathrm{C}^{+}, \mathrm{E}\right)$.

$$
P\left(f_{i} \mid C^{+}, E\right)=\frac{k\left(f_{i}, C^{+}, E\right)}{k\left(C^{+}, E\right)}
$$

$\mathrm{k}\left(\mathrm{C}^{+}, \mathrm{E}\right)$ represents the number of consumed items in the profile with a context equal to $\mathrm{E}$ and a positive user appreciation. A subset of these items is labeled with the feature $f_{i}$. The size of this subset is symbolized by $k\left(f_{i}, C^{+}, E\right)$. An analogous reasoning can be followed for $\mathrm{P}\left(\mathrm{f}_{\mathrm{i}} \mid \mathrm{C}^{-}, \mathrm{E}\right)$.

\section{ALGORITHM OPTIMIZATIONS}

To improve the efficiency of the proposed recommendation algorithm, the following optimizations can be applied.

\section{A. Eliminating Irrelevant Tags}

In contrast with strict classification systems (taxonomy), the metadata of user-generated content is usually less strict. (Relevant) keywords or tags get associated with or assigned to a piece of information. However, these folksonomies involve some extra difficulties by nature [13]: synonyms, subjective annotations, too general or irrelevant tags, etc.

Recent research on folksonomies has revealed that the most frequent occurring tags are too generic to be meaningful [25]. Examples are: 2008, funny, video, new, etc. Infrequent tags, which typically can be categorized as incidentally occurring words, such as mis-spellings and complex phrases, are also useless to incorporate in the viewer's profile. Our system eliminates these irrelevant tags to return the focus to the most informative keywords of the content item.

\section{B. Context Generalization}

The proposed context aware Bayesian classifier considers the consumption context, which consists of the time, the location, and the mood of the viewer, during the recommendation process.

$$
E=\{T, L, M\}
$$

In this way, variations in viewing preferences according to variations in the consumption context can be tracked. However, a partitioning of user history according to the consumption context, might lead to a profile fragmentation. If insufficient preference information for a specific context is available, for example because the user rarely utilizes the application in that context, the algorithm has to obtain its data from similar context situations. In this case, the 3dimensional context specification $E=\{T, L, M\}$ will be generalized (or relaxed) to a 2-dimensional context: $E^{\prime}=\{T, L\}, E^{\prime}=\{T, M\}$ or $E^{\prime}=\{L, M\}$. Further relaxation to a 1dimensional context, $E^{\prime \prime}=\{T\}, E^{\prime \prime}=\{L\}$ or $E^{\prime \prime}=\{M\}$, or even to a context without specifications, $E^{\prime \prime}=\{\}$, is possible.

A first case where a context relaxation can be necessary is during the calculation of $\mathrm{P}\left(\mathrm{C}^{+} \mid \mathrm{E}\right)$ and $\mathrm{P}\left(\mathrm{C}^{-} \mid \mathrm{E}\right)$ in (4) and (5). A denominator, $\mathrm{k}(\mathrm{E})$, below a predefined threshold, $\mathrm{T}_{\mathrm{E}}$, points to a profile with insufficient information about context environment $\mathrm{E}$. This will result in an unreliable estimation of the conditional probability and the associated content classification class. In this case, context relaxation will broaden the context and involve more data of the current user profile since:

$$
k\left(E^{\prime \prime \prime}\right) \geq k\left(E^{\prime \prime}\right) \geq k\left(E^{\prime}\right) \geq k(E)
$$

The conditional probability, calculated with the relaxed context will be estimated as follows: 


$$
P\left(C^{+} \mid E\right) \cong P\left(C^{+} \mid E^{\prime}\right)=\frac{k\left(C^{+} \mid E^{\prime}\right)}{k\left(E^{\prime}\right)}
$$

In the case that $k\left(E^{\prime}\right)$ is still below $T_{E}$, further relaxation will be performed until the threshold is reached. If $k\left(\mathrm{E}^{\prime \prime}\right)$ is reached, no further relaxation is possible since this term stands for all the consumptions in the user profile. If the value is still below the threshold, no reliable probability estimation can be made. This problem, where only a very sparse user profile is available, is commonly known as "the cold start problem" and is a worry for the majority of recommender systems.

Another motivation to expand the context before calculating $\mathrm{P}\left(\mathrm{C}^{+} \mid \mathrm{E}\right)$, is the avoidance of extreme probability values. A probability $\mathrm{P}\left(\mathrm{C}^{+} \mid \mathrm{E}\right)$, with a value of zero should correspond to a user which dislikes every content item in the specific context E. Conversely, a value of one for the probability should denote that the user likes every content item in that context. These two senseless situations, which are due to a lack of profile information in the selected context, might be corrected with context relaxation.

A second situation which may require context relaxation is the calculation of $\mathrm{P}\left(\mathrm{f}_{\mathrm{i}} \mid \mathrm{C}^{+}, \mathrm{E}\right)$ in $(8)$. Again, the denominator has to be above a predefined threshold, $\mathrm{T}_{\mathrm{C}+\mathrm{E}}$, to obtain a reliable estimation of the conditional probability. This means that the algorithm needs enough positive examples with a context environment equal to $\mathrm{E}$. The context might be relaxed until the threshold is reached or no further relaxation is possible (cold start problem).

An additional condition to obtain a reliable estimation of $\mathrm{P}\left(\mathrm{f}_{\mathrm{i}} \mid \mathrm{C}^{+}, \mathrm{E}\right)$ is that the profile contains sufficient examples of items, consumed in the context $\mathrm{E}$ and characterized by the feature $\mathrm{f}_{\mathrm{i}}$. In other words, $\mathrm{k}\left(\mathrm{f}_{\mathrm{i}}, \mathrm{E}\right)$ needs to be above a certain threshold, $\mathrm{T}_{\mathrm{fi}, \mathrm{E}}$. Once more, expanding the context environment $\mathrm{E}$, might provide the necessary information. If not, the viewer has not yet expressed a strong view for the feature $f_{i}$, and there is no means to estimate the probability accurately. Ignoring this feature and relying on the remaining features of the item for the content classification, can then be considered. The same reasoning is applicable for the calculation of $\mathrm{P}\left(\mathrm{f}_{\mathrm{i}} \mid \mathrm{C}^{-}, \mathrm{E}\right)$.

\section{Combining Multiple Generalized Contexts}

The goal of the context relaxation process is to acquire more user information by removing one of the features from the context specification. To preserve the most informative context, the least important feature should be removed from the context specification. For example the context, \{morning, at work, happy\}, may be generalized to \{?, at work, happy\} if the preferences do not vary (too much) over time, given the specified location and mood. The question mark in this notation indicates an unspecified feature of the context. However, it is not an easy task to determine which feature is the least important in a specific context, especially not if the context contains a large number of features. Moreover, the least important feature of the context can vary for every individual user.

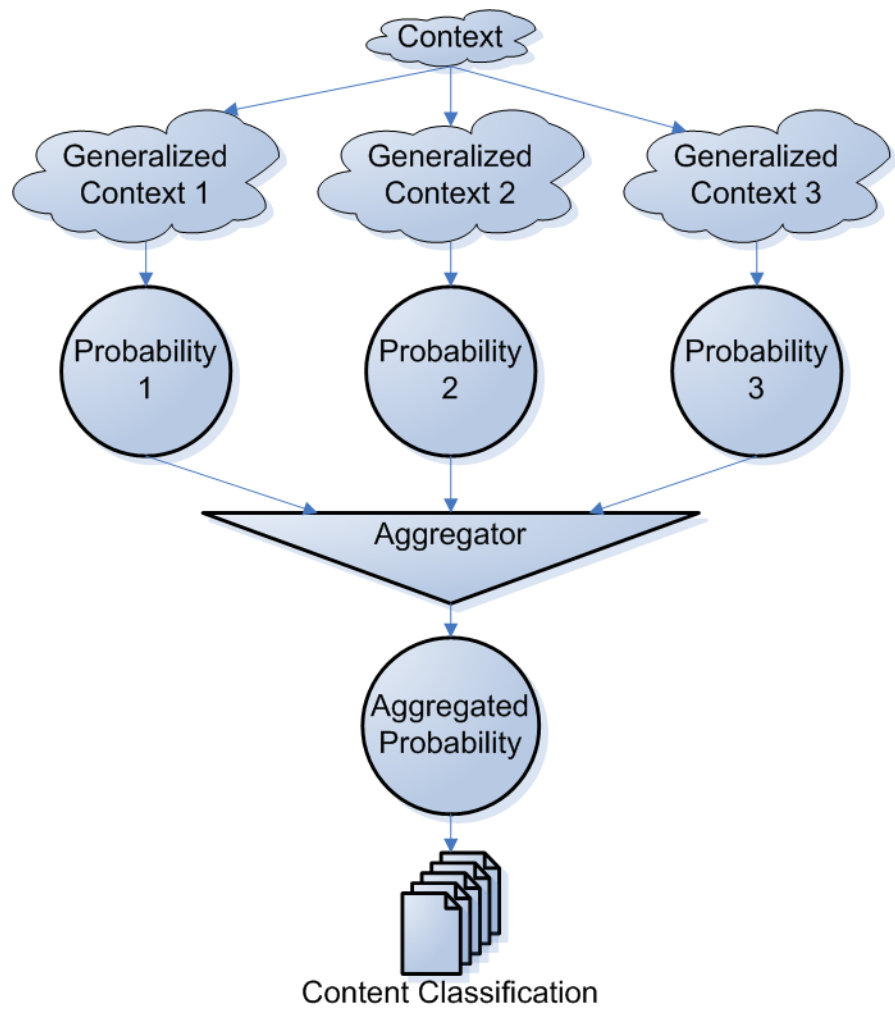

Figure 4. Aggregation of probabilities obtained by diverse generalized context specifications.

As a solution to this problem, we propose to relax a context repeatedly to all its possible generalizations. For example, the context $\mathrm{E}=\{\mathrm{T}, \mathrm{L}, \mathrm{M}\}$ will be generalized three times to a 2-dimensional context: $\mathrm{E}^{\prime}=\{\mathrm{T}, \mathrm{L}\}, \mathrm{E}^{\prime}=\{\mathrm{T}, \mathrm{M}\}$, and $\mathrm{E}^{\prime}=\{\mathrm{L}, \mathrm{M}\}$. The motivation behind this technique is that there can exist multiple relevant generalizations of a specific context. For example, the context \{morning, at work, ?\}, can be another relevant generalization of \{morning, at work, happy\}. Each of these generalized context specifications can be used to calculate the conditional probability, which is needed to classify content items. Finally, all the calculated probabilities will be combined and transformed in one conditional probability value by an aggregator. A simple design of an aggregator calculates for example a weighted average of the obtained conditional probabilities. This technique is illustrated in Figure 4.

\section{CONCLUSION AND FUTURE WORK}

In this paper, we have presented an improved version of the Bayesian classifier, together with the used profile structure and feedback mechanism, as a solution to the overabundance of user-generated content on mobile devices. This paper proofs that the Bayesian classifier allows the addition of the consumption context (time, location, mood of the user, etc.) to exploit the mobility and address the context dependent preferences of users. By relaxing the consumption context, the proposed algorithm relies on general user preferences for the early suggestions and will become more context specific as more user information becomes available. 
The next step in our research is evaluating the efficacy of the proposed contextual recommender. Traditional algorithm bench-marks for the quality of the recommendations operate on large data sets consisting of user preferences. However, according to our knowledge, no datasets with context information or mobile consumption preferences are publicly available. Therefore, the performance of the recommendations has to be evaluated by live experiments with human subjects. Unfortunately, this evaluation technique is time consuming, not reproducible and more labor intensive than an evaluation based on a dataset.

Furthermore, we will investigate how this context can best be incorporated in other traditional recommendation algorithms like collaborative filtering. Related to our work, a number of interesting research perspectives exist. Automatic retrieval of the environment (location, mood, etc) will release the user from the burden of specifying the context. Investigating the relevant contextual features could gain more insight in the content selection process: which context information is important for which type of application and how is it possible to adjust the suggestions to this context? Finally, the context can become a part of the recommendation itself. E.g., a location or a time can be recommended for a specific video.

\section{ACKNOWLEDGMENT}

We would like to thank the Research Foundation Flanders (FWO), for the research position of Toon De Pessemier (Aspirant FWO). We would also like to express our appreciation to the IBBT / UGent-INTEC (Interdisciplinary institute for BroadBand Technology / Ghent University - Department of Information Technology for supporting this research.

\section{REFERENCES}

[1] M. Cha, H. Kwak, P. Rodriguez, Y. Ahn, and S. Moon. "I tube, you tube, everybody tubes: analyzing the world's largest user generated content," In IMC '07: Proceedings of the seventh ACM SIGCOMM conference on Internet measurement, New York, NY, USA: ACM, pp. 1-14, 2007.

[2] "YouTube - Broadcast yourself," Feb 2005. Available at http://www.youtube.com 08.02.2010.

[3] "Vimeo, video sharing for you," Nov 2004. Available at http://www.vimeo.com 08.02.2010.

[4] "Digg - The lastest news headlines, videos and images," Nov 2004. Available at http://digg.com 08.02.2010.

[5] G. Linden, B. Smith, and J. York. "Amazon.com recommendations: item-to-item collaborative filtering," IEEE Internet Comput., vol.7, no.1, pp. 76-80, June 2003.

[6] G. Karypis. "Evaluation of item-based top-n recommendation algorithms," In CIKM '01: Proceedings of the tenth international conference on Information and knowledge management, New York, NY, USA: ACM, pp. 247-254, 2001.

[7] "Netflix dvd rental, movies streamed online, dvd \& blu-ray movie," 1997. Available at http://www.netflix.com 08.02.2010.

[8] S. Pfeiffer and U. Srinivasan, "TV anytime as an application scenario for MPEG-7," In Proceedings of the 2000 ACM workshop on Multimedia, pp. 89-92, 2000.
[9] H. Lee, S.-J. Yang, J.-G. Kim, and J. Hong. "Personalized TV services based on TV-anytime for PDR," Consumer Electronics, 2005. ICCE. 2005 Digest of Technical Papers. International Conference on, pp. 115-116, Jan. 2005.

[10] H. Zhang, S. Zheng, and J. Yuan, "A personalized TV guide system compliant with MHP," IEEE Trans. Consumer Electron., vol.51, no.2, pp.731-737, May 2005.

[11] K. Kurapati, S. Gutta, D. Schaffer, J. Martino, and J. Zimmerman, "A multi-agent TV recommender" In Proceedings of the UM 2001 workshop Personalization in Future TV, pp. 13-14, 2001.

[12] Z. Yu and X. Zhou. "TV3P: an adaptive assistant for personalized TV. Consumer Electronics", IEEE Trans. Consumer Electron., vol. 50, no. 1 pp.393-399, Feb 2004.

[13] S. Golder and B. A. Huberman, "The structure of collaborative tagging systems," ArXiv Computer Science e-prints, Aug. 2005.

[14] M. Szomszor, C. Cattuto, H. Alani, K. O'Hara, A. Baldassarri, V. Loreto, and V. D. Servedio, "Folksonomies, the semantic web, and movie recommendation," In 4th European Semantic Web Conference, Bridging the Gap between Semantic Web and Web 2.0, 2007.

[15] "Grouplens Research," Available at http://www.grouplens.org 08.02.2010.

[16] B. Miller, I. Albert, S. K. Lam, J. A. Konstan, and J. Riedl, "Movielens unplugged: experiences with a recommender system on four mobile devices," In Proceedings of the 2003 ACM Conference on Intelligent User Interfaces, Miami, FL, Jan. 2003.

[17] B. L. Tseng, C.-Y. Lin, and J. R. Smith, "Video summarization and personalization for pervasive mobile devices". In M. M. Yeung, C.-S. $\mathrm{Li}$, and R. W. Lienhart, editors, Society of Photo-Optical Instrumentation Engineers (SPIE) Conference Series, vol. 4676, pp. 359-370, Dec. 2001.

[18] P. Dominogos and M Pazzani, "On the optimality of the simple bayesian classifier under zero-one loss". In Machine Learning, vol. 29, no. 2-3, pp. 103-130, 1997

[19] J. Xu, L.-J. Zhang, H. Lu, and Y. Li, "The development and prospect of personalized TV program recommendation systems," In MSE '02: Proceedings of the Fourth IEEE International Symposium on Multimedia Software Engineering, Washington, DC, USA, pp. 82, 2002.

[20] Y. Blanco-Fernandez, J. J. Pazos-Arias, A. Gil-Solla, M. RamosCabrer, B. Barragans-Martinez, and M. Lopez-Nores, "A multi-agent open architecture for a TV recommender system: a case study using a Bayesian strategy," In ISMSE '04: Proceedings of the IEEE Sixth International Symposium on Multimedia Software Engineering, Washington, DC, USA, pp 178-185, 2004.

[21] J. Zimmerman, K. Kurapati, A. L. Buczak, D. Schaffer, S. Gutta, and J. Martino, "TV personalization system design of a TV show recommender engine and interface," in Personalized Digital Television, Kluwen Academic Publishers, The Netherlands, pp. 2753, 2004.

[22] A. Goker and H. I. Myrhaug, "User context and personalization," In ECCBR Workshop on Case Based Reasoning and Personalisation, Aberdeen, 2002.

[23] A. Zimmermann, M. Specht, and A. Lorenz, "Personalization and context management". In User Modeling and User-Adapted Interaction, vol. 15, no.3-4, pp. 275-302, 2005.

[24] P. Domingos and M. Pazzani, "Beyond independence: conditions for the optimality of the simple Bayesian classifier" In Machine Learning, Morgan Kaufmann, pp. 105-112, 1996.

[25] B. Sigurbjörnsson and R. van Zwol, "Flickr tag recommendation based on collective knowledge," In WWW '08: Proceeding of the 17 th international conference on World Wide Web, New York, NY, USA, ACM, pp 327-336, 2008. 